\title{
Pediatric safety: review of the susceptibility of children with disabilities to injuries involving movement related events
}

\author{
Abbey Fraser ${ }^{1 *} \mathbb{D}$, Dao Doan ${ }^{1}$, Mary Lundy ${ }^{2}$, Grant Bevill $^{1}$ and Juan Aceros ${ }^{1}$
}

\begin{abstract}
Background: Toy-related injuries have increased significantly in the past decade, in particular those related to rideon toys. This increase has been attributed to movement related events such as falls and inertial impacts.

Furthermore, children with disabilities have been reported to be at a greater risk of being injured, and are therefore more susceptible to toy-related injuries. Although, efforts are being made to modify ride-on toys as a method for increasing quality of life in children with disabilities, there are very limited pediatric safety studies regarding children with disabilities and modified ride-on toys.

Methods: This manuscript presents a systematic review of literature summarizing the current state of toy-related injuries including children with and without disabilities. Children exposed to inertial impacts in motor vehicle crashes have also been reviewed to present current pediatric safety testing methodologies and injury tolerance thresholds. Out of 2608 articles, 10 studies were included regarding current trends in toy-related injuries and safety testing methodologies.
\end{abstract}

Conclusions: From this study, a gap in the literature was discovered concerning the susceptibility of children with disabilities to toy-related injuries, specifically in relation to ride-on toys and the repercussion surrounding such injuries. It is theorized that such lack of data is due to the difficulty and costs associated with experimental validation. Hence, it is recommended that computer simulations be used to provide preliminary data analysis.

Keywords: Toy-related injuries, Ride-on toy, Pediatric safety, Disabilities, Collision, Children

\section{Background}

Pediatric safety had garnered more attention as of late in the scientific community (e.g. car seat safety, modified ride on toys for children with disabilities, etc.). Despite this recent interest, limitations exist in the knowledge of pediatric safety testing and tolerance thresholds due to a limited amount of test data. Children are unable to volunteer as test subjects and child cadavers are not readily available for research. Often, research is done using anthropomorphic test dummies that model the average child. This presents a problem however because it does not account for children with disabilities. Moreover, in recent years, children with disabilities have seen an increase in opportunities for transportation due to power

\footnotetext{
* Correspondence: fraserabbey3@gmail.com

${ }^{1}$ College of Computing, Engineering \& Construction, University of North

Florida, 1 UNF Dr, Jacksonville, FL 32224, USA

Full list of author information is available at the end of the article
}

mobility technologies and modified ride-on toys. These modified vehicles provide children with disabilities the chance to play and move in their environment.

This is a significant advancement for children, because toddlers and preschoolers require independent exploration of their environment for their brain cells and neural connections to develop properly (Thompson 2001). Around six months of age children will seek to move toward items that capture their interest and engage in independent, self-directed exploratory play (Thompson 2001). Toys help children participate in these activities and develop the necessary cognitive, social, and motor skills to manage a fulfilling life (Ginsburg 2007). This is true, and especially important, for children whose disabilities often keep them from engaging in normal play. As part of these independent, exploratory play activities, accidents are bound to happen and expected as part of their development. 
Pediatric safety research and regulation concentrates on protecting children from hazards such as toy-related injuries. It has been reported that the annual injury rate of toy-related injuries in children has increased from 1990 to 2011 by $39.9 \%$. This has been highly correlated to injuries caused by ride-on toys, as they account for $34.9 \%$ of all injuries (Gaw, Abraham, Gaw, Chounthirath, \& Smith 2015). Most injuries caused by ride-on toys are due to falls and inertial impacts. Furthermore, the number and rate of injuries have been reported to peak at 2 years of age -an age when children are still beginning to learn about their environment through movement and play-.

In recent years, researchers have engaged in the development of adaptive technology in the form of modified ride-on toys to provide children with disabilities independent self-directed mobility (Logan, S. W., Feldner, H. A., Galloway, J. C., \& Huang, H. 2016). It is noted that for this manuscript, a ride-on toy was defined as any rideable toy used for the purpose of play. Also, limited work has been done in studying the safety of such devices, and how modifications can worsen or improve the safety of a device in relation to a disability. Most studies have been conducted reviewing toy-related injuries in children without disabilities. One study comprehensively investigated toy-related injuries from a nationally representative data set collected from 1990 to 2011. This was the first time such a study was conducted. Mechanism of injuries were defined and separated into categories. Children were divided by age as well: 5 years of age or younger, and age 5 to 17 . Most injuries were found to be caused by ride-on toys and this number increased by $73.7 \%$ from 1990 to 2011 . It was hypothesized this is due to the increased popularity and accessibility of ride-on toys. These injuries spiked in 2000 and 2001. However, due to increased safety regulations, a noticeable decline in ride-on toy related injuries was observed. This displays the importance and impact of safer design and increased regulation.

A few other studies have investigated the susceptibility of children with disabilities to injury. One pooled data from the years 1997-2005 from the National Health Interview Survey, which is a multipurpose health survey completed annually by the United States Census Bureau, for the National Center for Health Statistics. To compare prevalence of injury between children with and without disabilities, a child with disabilities was matched to a healthy child of the same gender and age. It was determined that socioeconomic variables were insignificant. It was also found that children with disabilities experience a higher rate of injury $(3.8 \%$ vs $2.5 \%$; $P<.01)$. It was found that the risk of injury varied by the type of disability such that the more severe the disability, the higher the rate of injury.
Another study utilized the China Disabled Persons' Federation (CDPF) to conduct a study on all children with disabilities ages 1-14 (H. Zhu et al. 2014). The CDPF maintains a registry database that monitors the number of persons with disabilities and tracks the medical and rehabilitation services provided by the government. Each child with a disability was matched with a healthy child of same gender, age, and living in the same neighborhood for the study. Disabilities were categorized as vision, hearing, speech, physical disabilities, intellectual disabilities, and mental health disorders. Children were also organized by four levels of disabilities, defined as level four being most severe and nonfunctional, level three being less severe and minimal functionality, level two being semi-functional and level one being the mildest degree of disability with the best functionality. Sociodemographic variables were taken into consideration, including: gender and age of the child, parent's education, family income, single-parent family status, time of being supervised by an adult each day, and total number of family members (H. Zhu et al. 2014).

Injuries were assessed when an injured child sought medical care at a hospital or community clinic. The rate of injury among healthy children was found to be $4.4 \%$, but increased to $9.6 \%$ for children with a single disability, and $11.2 \%$ for children with multiple disabilities. The level of disability that was injured the most was level 2 (11.5\%), followed by level 3 (10.4\%), level 4 (10.3\%), and level 1 (8.1\%) (Klevberg, Elkjaer, Jahnsen, Elvrum, Zucknick, Ostensjo, \& Krumlinde-Sundholm 2018). Emerging evidence indicates that individuals with disabilities face a significantly higher risk of injury than those without disability.

\section{Systematic literature search and data extraction}

Two independent researchers performed a systematic literature search to identify all relevant studies pertaining to pediatric safety and toy-related injuries for children with and without disabilities. Due to the limited number of studies on toy safety for children with disabilities, the search was divided into two sections. Section 1 focused on toy-related injuries. Section 2 focused on inertial impact and injury risk in vehicular collisions. This information is relevant due to its relation to inertial impacts caused by ride-on toys.

The following search terms were used: pediatric OR children OR pediatric OR child OR infant AND injury OR injuries OR accident OR trauma AND disability OR disabilities OR disabled OR handicap for the first section. Search terms safety AND inertia impact OR crash or "car crash" AND ATDs OR cadavers OR "computer simulations" AND "low-speed" AND scaling AND scaling techniques AND crash analysis were added to the first search terms for the second section. 
All titles that were relevant to the criteria went through a subsequent screening based on their abstract, and full text articles were reviewed once they were determined appropriate for this study. Studies were excluded when: high velocity impact injuries were surveyed, there was insufficient data concerning children with disabilities, adult participants were included. Fig. 1 describes the process of search and screening.

\section{Results: Methodology employed in the studies Pediatric safety testing Safety testing by anthropometric test devices (ATDs)}

Two studies investigated child safety in motor vehicle impacts by conducting a series of sled tests with 6 and 10-year-old anthropometric test devices (ATDs) using $48-50 \mathrm{~km} / \mathrm{hr}$. frontal crash pulse. One study investigated injury risk of a pediatric occupant with a disability sitting in a wheelchair while being transported (Ha \& Bertocci 2007). The other studied the possibilities of better protection when children were not using booster seats. Methods of increasing protection that were studied include various cushion lengths and varying lap belt geometry (Rosenbaum \& Rosenbloom 2012). Table 1 shows an overview of the studies.
Data related to head, chest, and pelvis acceleration, femur and neck forces, chest compression, chest deflection, and moment were measured during each test. The results from the wheelchair occupant study were then compared with the kinematic limitations and injury criteria of the Federal Motor Vehicle Safety Standards (FMVSS). Comparison to FMVSS 213 (Safety standard that must be met for children car seats to be sold for use. Includes requirements such as the child restraint system must pass a 30 miles per hour frontal sled test that simulates a crash, padding requirements, flammability standards, and buckle release pressure.) and FMVSS 208 (Safety standard for occupant crash protection that establishes performance requirements for passenger vehicles (Lewandowski 2006)) was used to determine the injury risk of the pediatric wheelchair occupant in a motor vehicle crash (Ha \& Bertocci 2007).

The study that investigated alternative seat belt protection used head excursion, peak knee excursion, the difference between peak head and peak knee excursion, and maximum torso angle to determine whether sitting with a shorter cushion and mid or forward angle lap belt would be better for safety when children are not using booster seats.
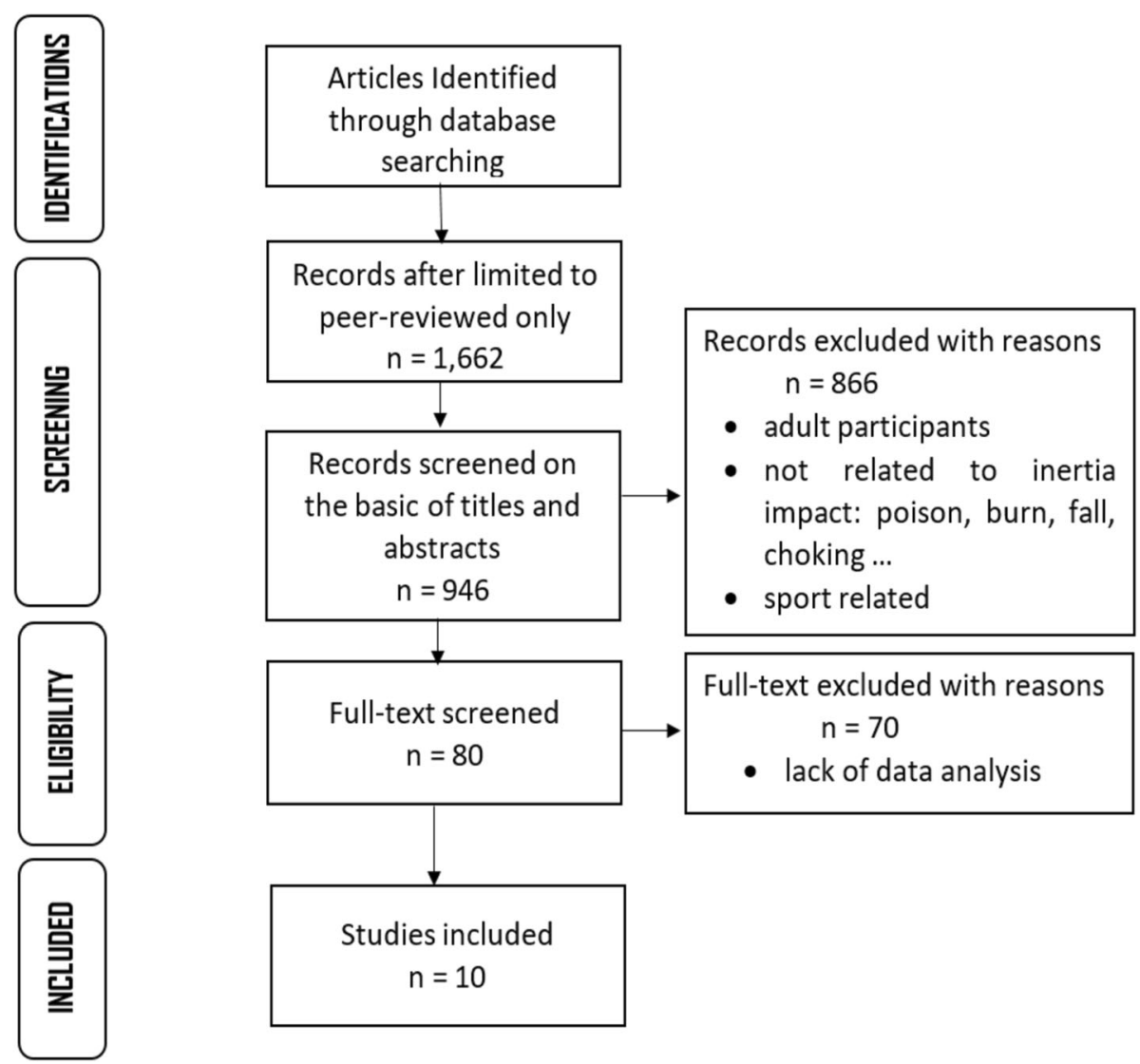

Fig. 1 Search process and identification of relevant studies 


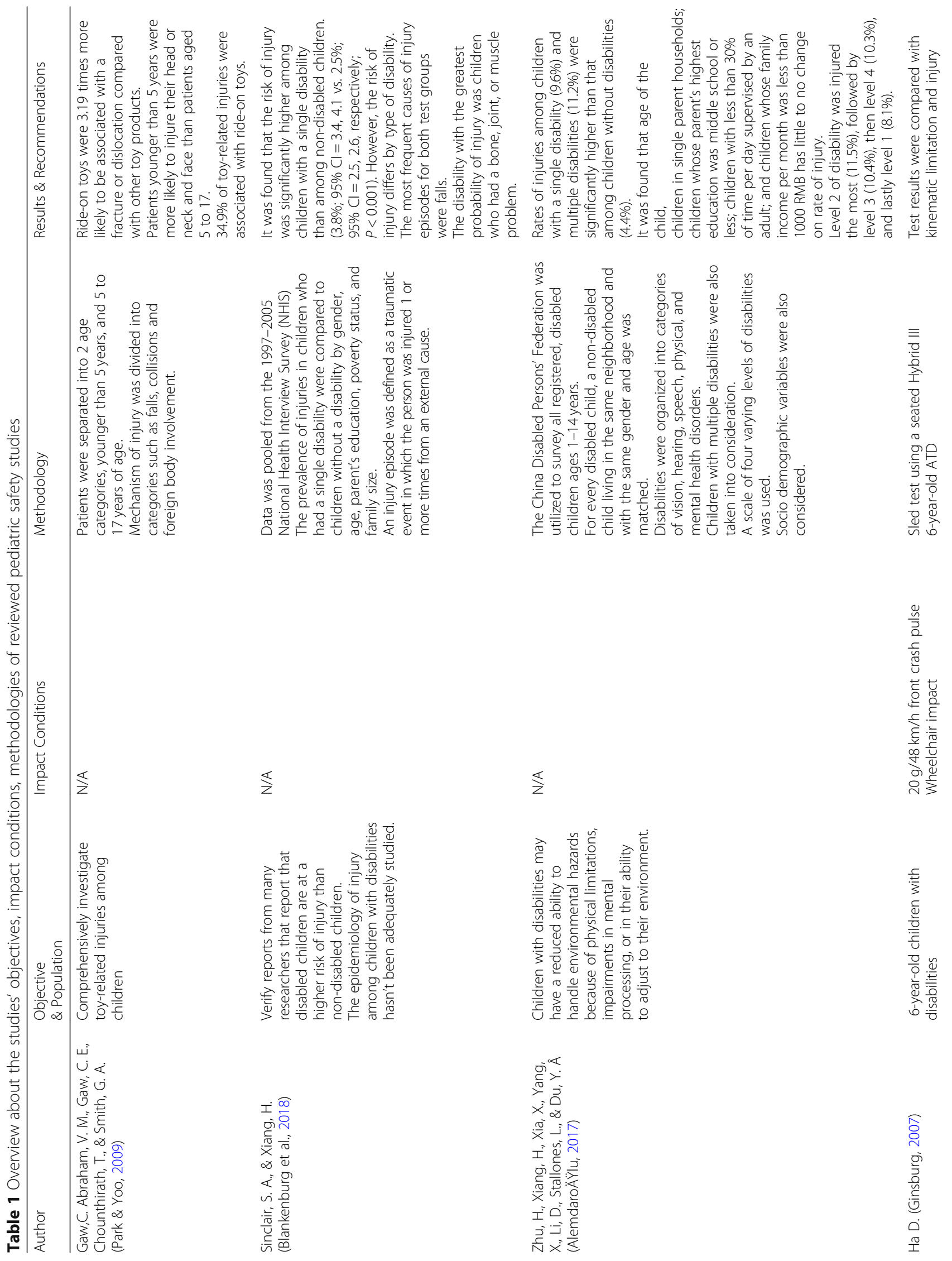




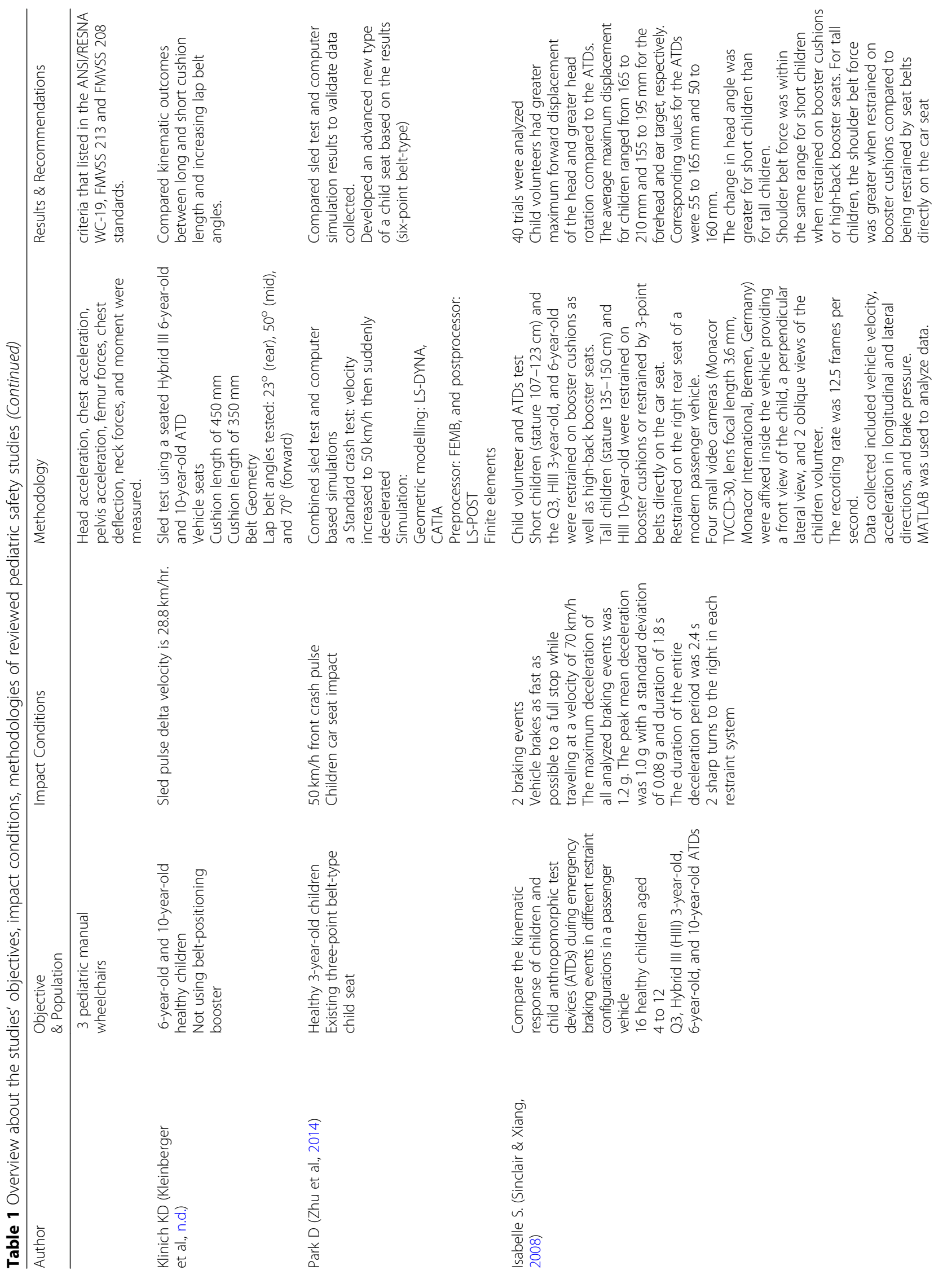




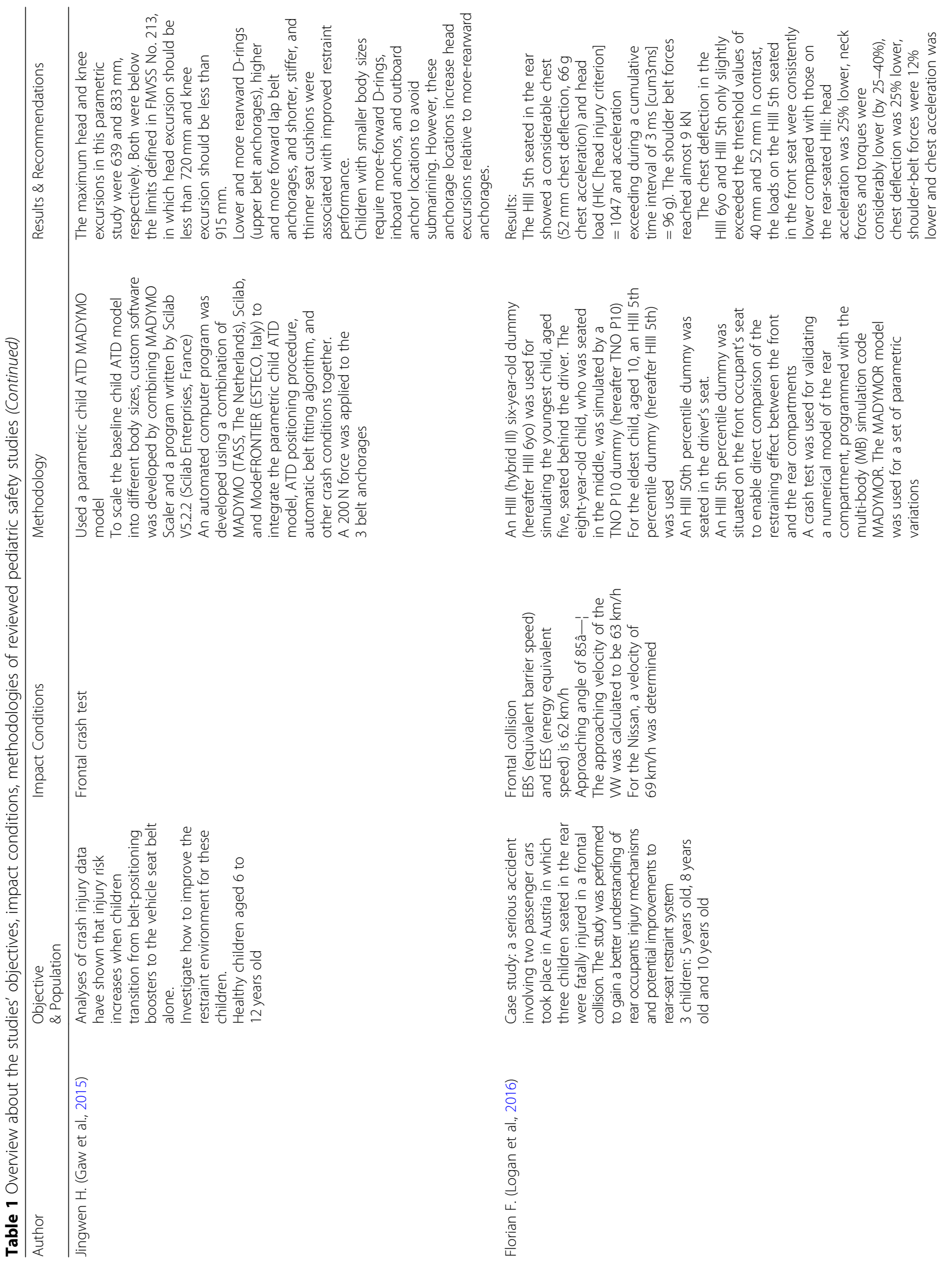




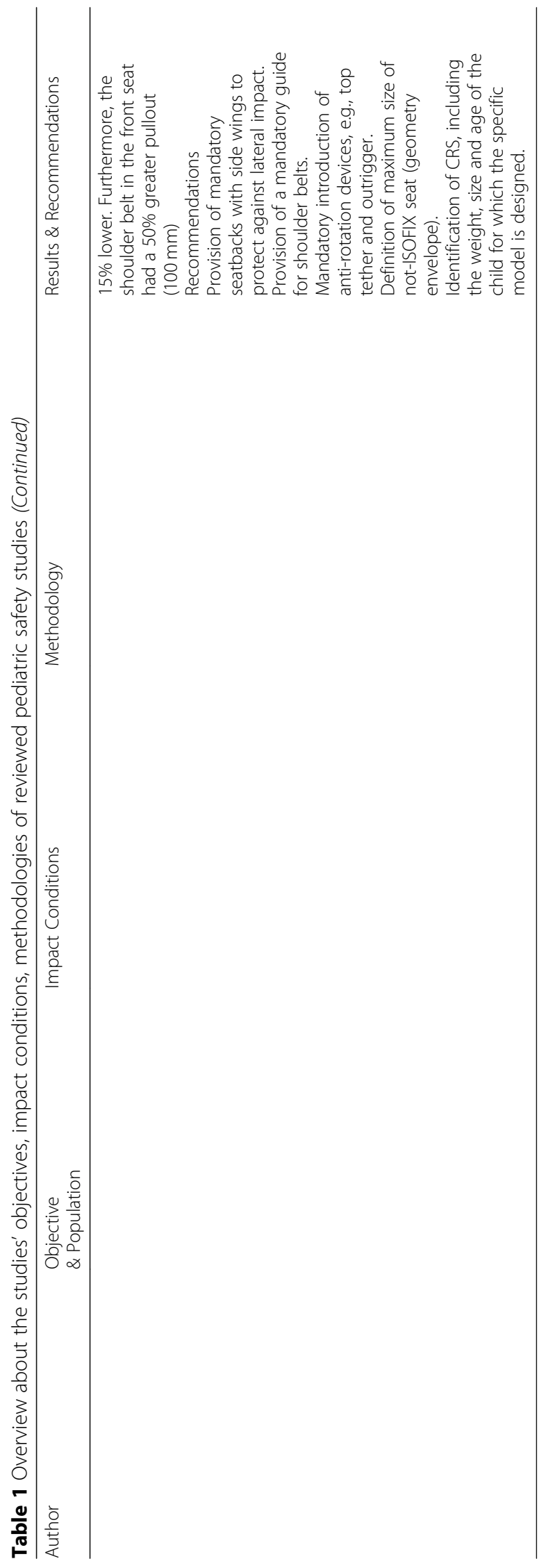




\section{Safety testing by ATDs and computer simulations}

One study used the combination of sled testing and computer simulations to develop an advanced child restraint system (CRS) (Park \& Yoo 2009).

A sled test was first performed using a 3-year-old ATD in an existing three-point seat belt CRS with the objective of achieving head and chest accelerations within safety limits. The crash test was designed to exert accelerations according to national standards, and increased velocity to $50 \mathrm{~km} / \mathrm{h}$ and then suddenly decelerated. A dynamic simulation was then conducted using a commercial LS-DYNA ${ }^{\circ}$ program developed by Livermore software Technology Corporation. LS-DYNA ${ }^{\bullet}$ was used for contact and collisions, and the computer-aided three-dimensional interactive application $\left(\right.$ CATIA $^{\mathrm{Tm}}$ ) program was used for geometric modeling. Once the sled test and computer simulation results was matched, a new type of child seat was developed. An optimization sequence was applied to determine the thickness of each part to decrease the weight. A new six-point CRS was then developed using LS-DYNA. Once the final result was obtained from the computer simulation of the new design, the sled test was carried out with the developed prototype of a six-point child seat (Park \& Yoo 2009). An overview is shown in Table 1.

\section{Results: Main findings of the studies Pediatric safety testing Safety testing by anthropometric test devices (ATDs)}

Sled tests were conducted under $48 \mathrm{~km} / \mathrm{h}$ and $20 \mathrm{~g}$ average impact conditions on children riding in a motor vehicle while seated in a wheelchair. This study showed that a 6-year-old seated in a wheelchair may be at risk of neck injury during a frontal car crash and concluded that variations in the shoulder belt anchor point led to variance in restraint effectiveness. All tests conducted in this study exceeded the tension extension limit. All tests complied with the requirement that the wheelchair not load the ATD. None of the tests exceeded the limit which evaluates the integrity of seat surface and seat attachment hardware and none of the tests exceeded the maximum chest acceleration limit of $60 \mathrm{~g}$. The results of the safety testing satisfied the head injury criterion (HIC) of 700 which measures the amount of damage to the head. Chest deflection for two iterations of the test were at the limit of $40 \mathrm{~mm}$ specified in regulation. The first and second sled test exceeded the peak neck tension force limit of $1490 \mathrm{~N}$. No tests exceeded the independent compressive neck force limits (Ha \& Bertocci 2007).

When cushion size and lap belt angle were tested, increased cushion length and bigger lap belt angles improved children safety in a motor crash. However, seat boosters still have the best child safety performance than simply increasing the cushion length and lap belt angle.

\section{Safety testing by ATDs and computer simulations}

While the study failed to match the sled test results to simulations results exactly, the collected data and magnitudes at the peak value were comparable. Based on the resulting similar trends, it was concluded that the simulation sequence was suitable to develop a new child seat.

The design of a six-point belt-type child seat was carried out resulting in a lightweight design to save material and manufacturing cost. However, such a lightweight design compromises the safety of the seat. Simulations varying the thickness of the material was carried out using ANSYS (A computer simulation software) to explore proper thickness vs safety tradeoffs. Results yielded a final design having $64.5 \%$ of its original volume. Once computer simulations were performed for the new six-point CRS, the sled test was carried out indicating that a six-point CRS provides a lower impact force due to the force being distributed over an increased area. (Park \& Yoo 2009).

\section{Discussion}

Pediatric safety testing

Safety testing by anthropometric test devices (ATDs)

Children with disabilities often differ anatomically from children without disabilities, and therefore are often required to remain seated in their wheelchair while being transported in a motor vehicle. Injury risk of a child, seated in a manual pediatric wheelchair, was analyzed in this study using frontal impact sled testing. A 6HybridIII ATD was used, which models a non-disabled 6-year-old child with normal muscle tone and balance. Therefore, a child with disabilities may be more susceptible to severe and fatal injuries in circumstances where a child without disabilities would not be injured (Ha \& Bertocci 2007).

This study showed that a 6-year-old seated in a wheelchair may be at risk of neck injury during a frontal car crash. The study also concluded that variation in the shoulder belt anchor point led to variance in restraint effectiveness. It was hypothesized that chest deflections would have been higher if the shoulder belt had been at a more optimum anchorage point (Ha \& Bertocci 2007).

\section{Safety testing by ATDs and computer simulations}

Dynamic simulations of a child seat were carried out using LS-DYNA ${ }^{\circ}$ to develop an advanced CRS design. Simulation results for a six-point belt-type child seat were compared to sled testing concluding that LS-DYNA $^{\circ}$ is a suitable alternative to replace sled testing, reducing cost and time for new product development (Park \& Yoo 2009). However, it is acknowledged in this study that precise material properties are needed for accurate results. 


\section{Conclusion}

Studies have been carried out on the multiple aspects of toy-related injuries and the susceptibility of children with disabilities to injury. However, a gap in the literature occurs concerning the susceptibility of children with disabilities to toy-related injuries, specifically in relation to ride-on toys and the repercussions surrounding such injuries. It is theorized that such lack of data is due to the difficulty and costs associated with experimental validation. Hence, it is recommended that computer simulations be used to provide preliminary data analysis. Various aspects of small inertial impacts on a child with disabilities could be drawn from these studies. Furthermore, safety recommendations for ride-on toy modifications could be derived from such simulations and these could be correlated to specific disabilities. Ultimately the goal of such work would be to draw specific guidelines regarding modifications of ride-on toys and children with disabilities.

\section{Abbreviations}

ATDs: Anthropometric test devices; CATIA ${ }^{\mathrm{TM}}$ : Computer-aided threedimensional interactive application; CDPF: The China Disabled Persons' Federation; CRS: Child restraint system; FMVSS: Federal Motor Vehicle Safety Standards; HIC: Head injury criterion

\section{Acknowledgements}

None.

\section{Funding}

This work was supported by the Eunice Kennedy Shriver National Institute of Child Health \& Human Development of the National Institutes of Health under Award Number R25HD094335. The content is solely the responsibility of the authors and does not necessarily represent the official views of the National Institutes of Health.

\section{Availability of data and materials}

The datasets used and/or analyzed during the current study are available online or available from the corresponding author on reasonable request.

\section{Authors' contributions}

JA and ML conceived the study. JA designed the study. AF and DD performed the data collection, and performed the data analysis. AF performed the interpretation of the data and drafted the manuscript. JA, ML and GB provided critical revisions, administrative support, and final approval of the submitted manuscript. All authors read and approved the final manuscript.

\section{Ethics approval}

This article does not contain any studies with human participants or animals performed by any of the authors.

\section{Consent for publication}

All the participants provided consent for publication.

\section{Competing interests}

Abbey Fraser declares that she has no conflict of interest. Dao Doan declares that she has no conflict of interest. Mary Lundy declares that she has no conflict of interest. Grant Bevill declares that he has no conflict of interest. Juan Aceros declares that he has no conflict of interest. The authors declare that they have no competing interests.

\section{Publisher's Note}

Springer Nature remains neutral with regard to jurisdictional claims in published maps and institutional affiliations.

\section{Author details}

${ }^{1}$ College of Computing, Engineering \& Construction, University of North Florida, 1 UNF Dr, Jacksonville, FL 32224, USA. ${ }^{2}$ Brooks College of Health, University of North Florida, 1 UNF Dr, Jacksonville, FL 32224, USA.

Received: 21 November 2018 Accepted: 28 February 2019

Published online: 08 April 2019

\section{References}

Alemdaroğlu E, Öbudak SD, Mandiroğlu S, Biçer SA, Ã-zgirgin N, UÃßan H. Predictive factors for inpatient falls among children with cerebral palsy. J Pediatr Nurs 2017;32:25-31.

Blankenburg M, Junker J, Hirschfeld G, Michel E, Aksu F, Wager J, et al. Original article: quantitative sensory testing profiles in children, adolescents and young adults (6-20 years) with cerebral palsy: hints for a neuropathic genesis of pain syndromes. Eur J Paediatr Neurol. 2018;22:470-81.

Gaw C, Abraham VM, Gaw CE, Chounthirath T, Smith GA. Toy-related injuries among children treated in US emergency departments, 1990-2011. Clin Pediatr. 2015;54(2):127-37.

Ginsburg KR. Committee on communications, and committee on psychosocial aspects of child and family health. The importance of play in promoting healthy child development and maintaining strong parent-child bonds. Pediatrics. 2007 Jan 1;119(1):182

Ha DR, Bertocci G. Injury risk of a 6-year-old wheelchair-seated occupant in a frontal motor vehicle impact-'ANSI/RESNA WC-19' sled testing analysis. Med Eng Phys. 2007;29(7):729-38.

Kleinberger M, Sun E, Eppinger R, Kuppa S, Saul R. Development of Improved Injury Criteria for the Assessment of Advanced Automotive Restraint Systems. 1998 September,

Klevberg GL, Elkjaer S, Jahnsen R, Elvrum AG, Zucknick M, Ostensjo S, et al. Development of bimanual performance in young children with cerebral palsy. Dev Med Child Neurol. 2018;60(5):490-7.

Lewandowski J. Federal Motor Vehicle Safety Standard (FMVSS) 208 - occupant crash protection: right front passenger test methodologies. Apr. 2006;3.

Logan SW, Feldner HA, Galloway JC, Huang H. Modified ride-on Car use by children with complex medical needs. Pediatr Phys Ther. 2016;28(1):100-7.

Park D, Yoo W. A study on the design of a child seat system with multipoint restraints to enhance safety. Journal of Mechanical Science \& Technology. 2009;23(12):3316.

Rosenbaum P, Rosenbloom L. Cerebral palsy : from diagnosis to adult life. London: Mac Keith Press; 2012.

Sinclair SA, Xiang H. Injuries among US children with different types of disabilities. Am J Public Health. 2008;98(8):1510-6.

Thompson RA. Development in the first years of life. Futur Child. 2001;11(1):21-33.

Zhu H, Xiang H, Xia X, Yang X, Li D, Stallones L, et al. Unintentional injuries among Chinese children with different types and severity of disability. Ann Epidemiol. 2014;24(1):23-8. 